\title{
The meta and bioinformatics analysis of fascin expression in gastric cancer: a potential marker for aggressiveness and worse prognosis
}

\author{
Hua-Chuan Zheng ${ }^{1}$ and Shuang Zhao ${ }^{1}$ \\ ${ }^{1}$ Department of Experimental Oncology and Animal Center, Shengjing Hospital of China Medical University, Shenyang \\ 110004, China \\ Correspondence to: Hua-Chuan Zheng, email: zheng_huachuan@hotmail.com \\ Keywords: fascin; gastric cancer; meta analysis; bioinformatics analysis \\ Received: June 01, $2017 \quad$ Accepted: September 08, $2017 \quad$ Published: November 06, 2017 \\ Copyright: Zheng et al. This is an open-access article distributed under the terms of the Creative Commons Attribution License 3.0 \\ (CC BY 3.0), which permits unrestricted use, distribution, and reproduction in any medium, provided the original author and source \\ are credited.
}

\section{ABSTRACT}

Fascin is a FSCN1-encoded actin bundling protein, and positively associated with proliferation, migration and metastasis of malignancies. Here, we performed a systematic meta and bioinformatics analysis through multiple online databases up to March 14, 2017. We found up-regulated fascin expression in gastric cancer, compared with normal mucosa $(p<0.05)$. Fascin expression was positively with lymph node metastasis, TNM staging and worse prognosis of gastric cancer $(p<0.05)$. According to bioinformatics database, FSCN1 mRNA expression was higher in gastric cancer than normal tissues $(p<0.05)$. According to Kaplan-Meier plotter, we found that a higher FSCN1 expression was negatively correlated with overall and progression-free survival rates of all cancer patients, even stratified by aggressive parameters $(p<0.05)$. These findings indicated that fascin expression might be employed as a potential marker to indicate gastric carcinogenesis and subsequent progression, even prognosis.

\section{INTRODUCTION}

Fascin is a $55 \mathrm{kDa}$ monomeric actin filament bundling protein originally isolated from sea urchin egg, and contributes to increased proliferation, altered $\beta 1$ integrin distribution, enhanced invasive capacity and dedifferentiation status $[1,2]$. Fascin can directly interact with the microtubule cytoskeleton, and control fascindependent focal adhesion dynamics and cell migration speed, which is due to fascin-FAK-src complex formation [3]. However, E3 ligase Smurf1 monoubiquitinates fascin at Lys247 and Lys250, which decreases the fascin bundling EC50, delays the initiation of bundle assembly, and accelerates the disassembly of existing bundles [4].

Fascin protein is critical for TGF $\beta$-induced invasion and filopodia formation in spindle- shaped tumor cells through the canonical Smad-dependent pathway $[5,6]$. GATA3 abrogates Smad4 -mediated fascin overexpression, invadopodium formation, and invasion of breast cancer cells by abolishing the interaction between
Smad4 and its DNA binding elements [7]. Snyder et al. [8] found that Stat $3-\mathrm{NF}-\kappa \mathrm{B}$ complex was necessary for fascin expression in metastatic breast cancer cells in response to IL-6 and TNF- $\alpha$. The prometastatic RSK2-CREB pathway increased fascin expression to promote tumor metastasis $[9,10]$. Osanai et al. [11] demonstrated that CYP26A1 up-regulated fascin, and subsequently enhanced cell apoptotic resistance, anchorage-independent growth, mobility, invasion and escaped premature senescence in breast cancer cells.

Conditional expression of fascin decreased mice survival and increased tumor burden compared to control animals, and fascin expression accelerated tumor progression and formation of invasive adenocarcinoma in adult tumor-bearing animals [12]. FCSN1- deficient KRas(G12D) p53(R172H) Pdx1-Cre mice had longer survival times, delayed onset of pancreatic ductal adenocarcinoma (PDAC), and a lower PDAC tumor burdens than KPC mice [13]. Fascin is widely expressed in the mature dendritic cells, mesencymal cells, endothelial 
cells and neurons of the human, but low or absent in adult epithelia. Recent data have highlighted that fascin is upregulated in many human cancer, and correlated with the clinical aggressiveness and poor patient survival [14, 15]. In the present study, we performed both meta- and bioinformatics analyses to clarify clinicopathological and prognostic significances of fascin expression in gastric carcinogenesis and subsequent progression.

\section{RESULTS}

\section{Characteristics of eligible studies}

As shown in Figure 1 and Table 1, a total of 11 articles on the relationship between fascin expression and cancer risk, clinicopathological or prognostic parameters of gastric cancer were retrieved for our meta-analysis by immunohistochemistry in PubMed, Web of Science, BIOSIS, SciFinder and CNKI. Only 7 articles contained the samples of normal gastric mucosa [16-22]. There appeared the comparison between fascin expression and clinicopathological characteristics of gastric cancer in 11 studies, including sex, depth of invasion, lymph node metastasis, TNM staging and Lauren's classification [1626].

\section{Association between fascin expression and cancer susceptibility of gastric mucosa}

We analyzed the association between fascin expression and cancer susceptibility of gastric normal mucosa in 7 studies with 1105 cancers and 667 controls. As a result, we found up-regulated fascin expression in gastric cancer, compared with normal mucosa $(\mathrm{p}<0.0001$, Figure 2A).

\section{Association between fascin expression and clinicopathological parameters of gastric cancer}

As shown in Figure 2B, there was no difference in fascin expression between male and female patients with gastric cancer $(\mathrm{p}>0.05)$. Fascin expression was not associated with $\mathrm{T}$ staging of gastric cancer (Figure 2C, $\mathrm{p}>0.05)$. A higher fascin expression was detected in gastric cancer with than with out lymph node involved (Figure $2 \mathrm{D}, \mathrm{p}<0.0007)$. Fascin expression was positively linked to TNM staging regardless of subgrouping methods (Figures $2 \mathrm{E}$ and $2 \mathrm{~F}, \mathrm{p}<0.05)$. Intestinal-type carcinoma showed similar level of fascin expression to diffuse-type one (Figure 2G, $\mathrm{p}>0.05$ ).

\section{Association between fascin expression and survival rate of gastric cancer}

As indicated in Figure $2 \mathrm{H}$, the pooled results from 3 studies demonstrated a significant association between fascin expression and overall survival in the patients with gastric cancer $(\mathrm{HR}=0.67,95 \% \mathrm{CI}: 0.48-0.93$, $\mathrm{p}=0.02$ ). Results showed that fascin overexpression had an unfavorable prognostic value in gastric cancer patients.

\section{Publication bias}

The heterogeneity test was performed as shown in Figure 3. Sensitivity analysis was used to evaluate individual study's influence on the pooled results by deleting one single study each time from pooled analysis. As a result, T-staging result of fascin expression in Tsai's study had a significant effect on the pooled OR. When this study was excluded, the heterogeneity test was significantly reduced (data not shown).

\section{The clinicopathological and prognostic significance of FSCN1 expression in gastric cancers}

Then, we used Cho's, DErrico's, Cui's and Wang's datasets to perform bioinformatics analysis and found that FSCN1 expression was higher in gastric cancer than normal tissues, even stratified into intestinal-, diffuse- and mixed-type carcinoma (Figure 4A, $\mathrm{p}<0.05$ ). According to Kaplan-Meier plotter, we found that a higher FSCN1 expression was positively correlated with overall and progression-free survival rates of all cancer patients, even stratified by gender, TNM staging, lymph node involvement, any treatment (i.e. surgery alone, 5-FUbase adjuvant and other), Lauren's classification and Her2 expression (Figure 4B and Table 2, $\mathrm{p}<0.05$ ). It was the same for the patients with M0, moderately- differentiated, perforation-negative, T2 or T3 cancer (Table 2, p<0.05). T4 or poorly- differentiated cancer patients with high FSCN1 expression showed a low progression-free survival time than those with its low expression $(\mathrm{p}<0.05)$.

\section{DISCUSSION}

Fascin overexpression was found to promote the proliferation, migration, and invasion of cholangiocarcinoma cells [27]. Darnel et al. [28] found that fascin silencing increased cell adhesive properties, decreased tumor growth, and cell motility and invasiveness, and drastically prevented the formation of lymph node metastases in prostate cancer cells. Further investigation showed that fascin up-regulated NF- $\kappa$ B activity, uPA, MMP-2 and MMP-9 expression, but down-regulated the expression and nuclear translocation of BRMS1, resulting in a higher ability of breast cancer cells to migrate and invade [29]. In oral squamous cell carcinoma, fascin overexpression led to significant increase in cell migration, cell invasion, and MMP-2 activity with increased levels of phosphorylated Akt, ERK1/2 and JNK1/2 [30]. Liang et al. [31] indicated that 
Table 1: Main characteristics of eligible studies

\begin{tabular}{|c|c|c|c|c|c|c|c|c|c|}
\hline First author & Year & Country & Ethnicity & $\mathbf{A S}$ & Cases & Control & Risk to cancer & Outcome & Quality \\
\hline Hashimoto Y & 2003 & Japan & Asian & Dako & 214 & & & Negative & 8 \\
\hline Tsai WC & 2007 & China & Asian & Neomarker & 100 & & & Negative & 8 \\
\hline Li XH & 2008 & Japan & Asian & Neomarker & 509 & 138 & $\mathrm{Up}$ & Negative & 9 \\
\hline Kim SJ & 2012 & China & Asian & Dako & 471 & & & Negative & 8 \\
\hline $\mathrm{Tu} \mathrm{L}$ & 2016 & China & Asian & Cell Signaling & 204 & 204 & Up & Negative & 9 \\
\hline Li K & 2009 & China & Asian & Dako & 76 & 76 & $\mathrm{Up}$ & & 8 \\
\hline Peng LT & 2011 & China & Asian & Neomarker & 52 & 52 & $\mathrm{Up}$ & & 8 \\
\hline Gan FL & 2013 & China & Asian & Neomarker & 67 & 67 & $\mathrm{Up}$ & & 8 \\
\hline Rao P & 2013 & China & Asian & Neomarker & 150 & & & Negative & 8 \\
\hline Li M & 2014 & China & Asian & Boster & 90 & 90 & Up & & 8 \\
\hline Li S & 2015 & China & Asian & Maxin & 107 & 40 & Up & & 8 \\
\hline
\end{tabular}

fascin promoted the growth and migration of non-small cell lung cancer (NSCLC) cells by activating YAP/TEAD signaling. To investigate the clinicopathological and prognostic significances of fascin expression, we analyzed
11 studies, which met specific inclusion criteria and had moderate to high quality according to their NOS scores.

Chen et al. [32] found that the levels of autoantibody against fascin in the patients with esophageal squamous carcinoma (ESCC) were significantly higher than in

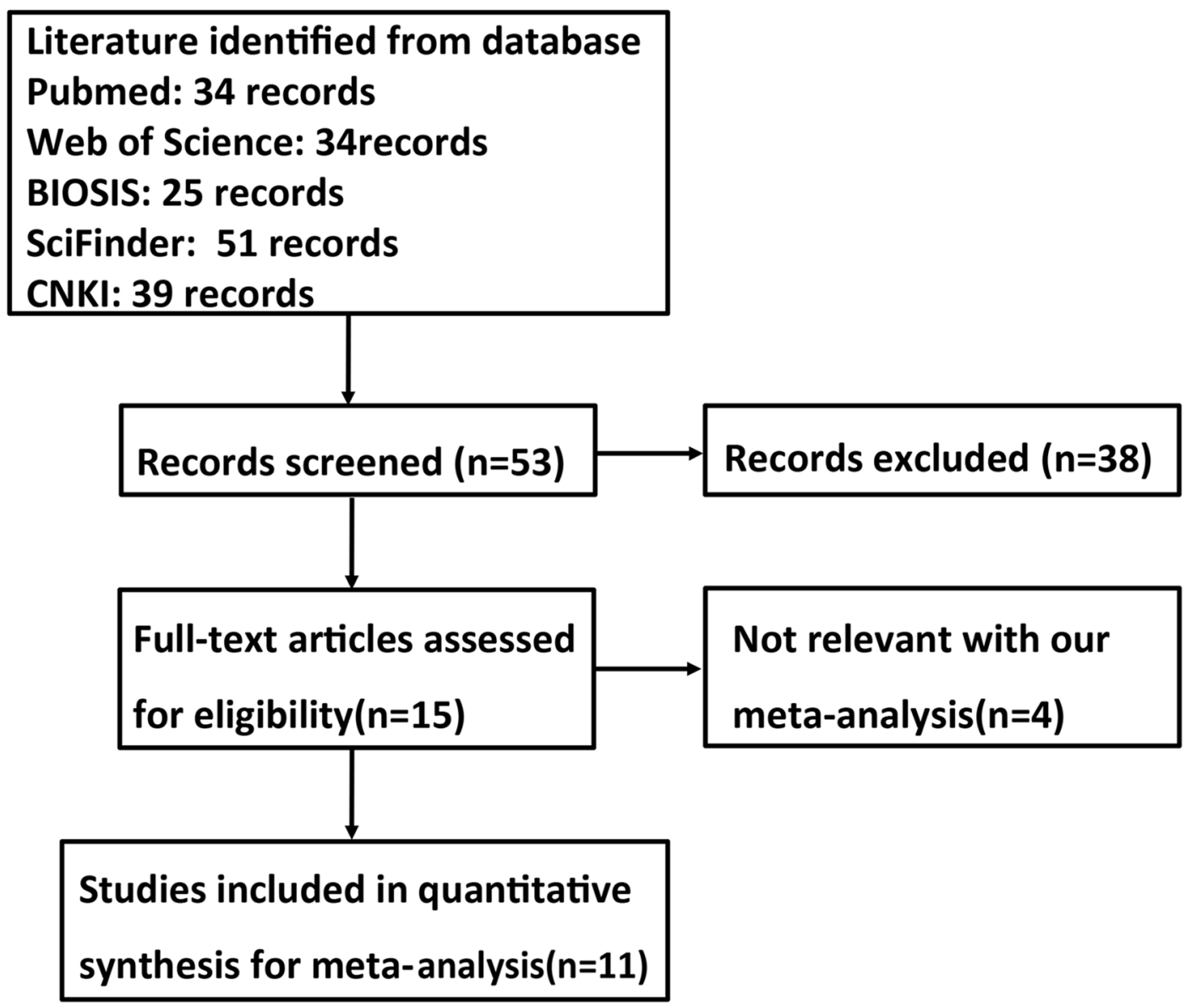

Figure 1: Flow diagram of the selection process in this meta-analysis. 


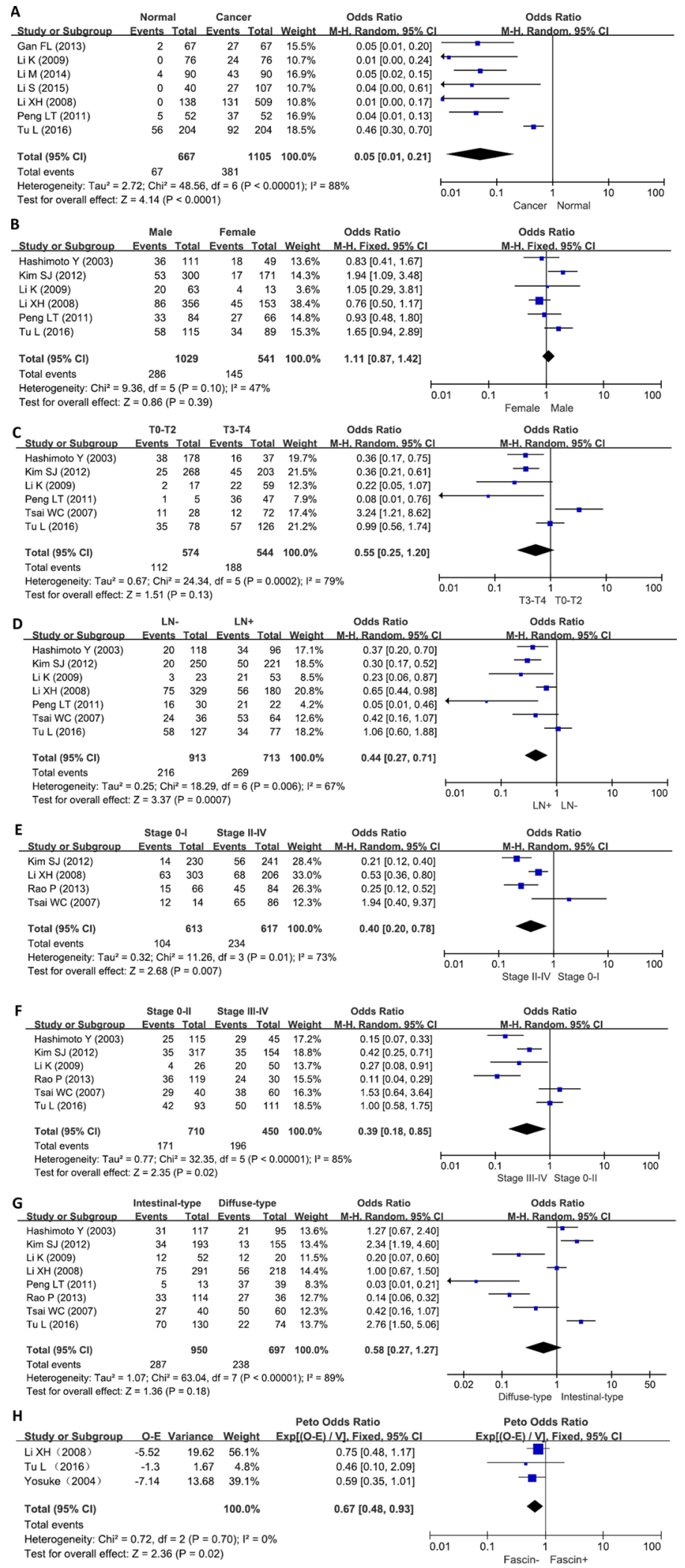

Figure 2: Forest plot for the relationship between fascin expression and clinicopatholoiocal parameters of gastric cancer. (A) gastric carcinogenesis (cancer vs normal mucosa); (B) correlation between sex and fascin expression (male vs female); (C) correlation between depth of invasion and fascin expression (Tis-2 vs T3-4); (D) correlation between lymph node metastasis (LN) and fascin expression (LN- vs LN+); (E) correlation between TNM staging and fascin expression (0-I vs II-IV); (F) correlation between TNM staging and fascin expression (0-II vs III-IV); (G) correlation between differentiation and fascin (intestinal-type vs diffuse-type); (H) correlation between survival rate and fascin expression (fascin - vs fascin + ). 
A

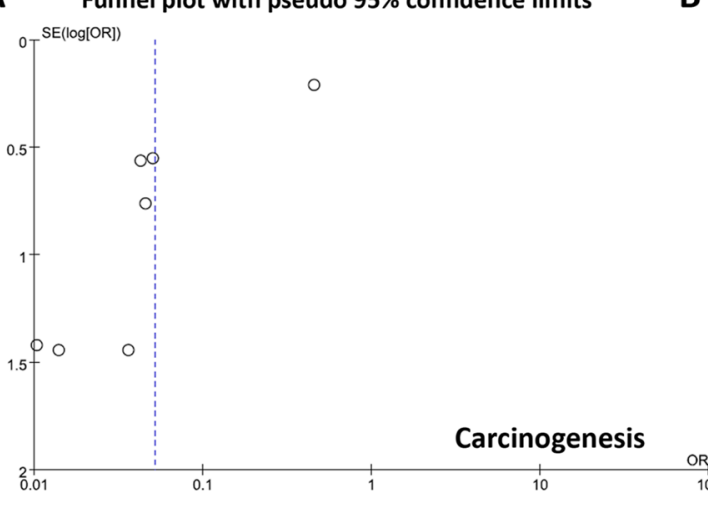

C

Cunnel plot with pseudo 95\% confidence limits

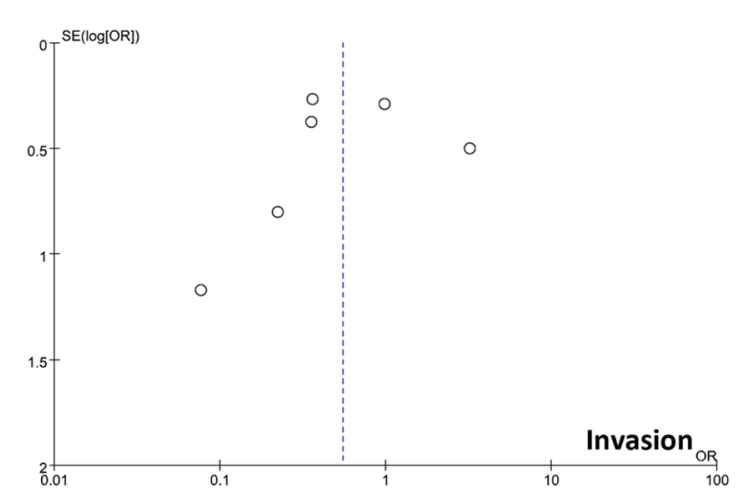

$\mathbf{E}$

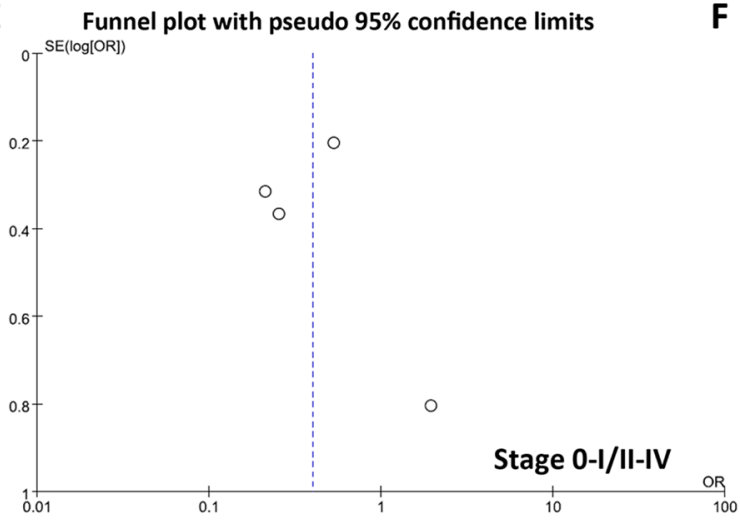

G

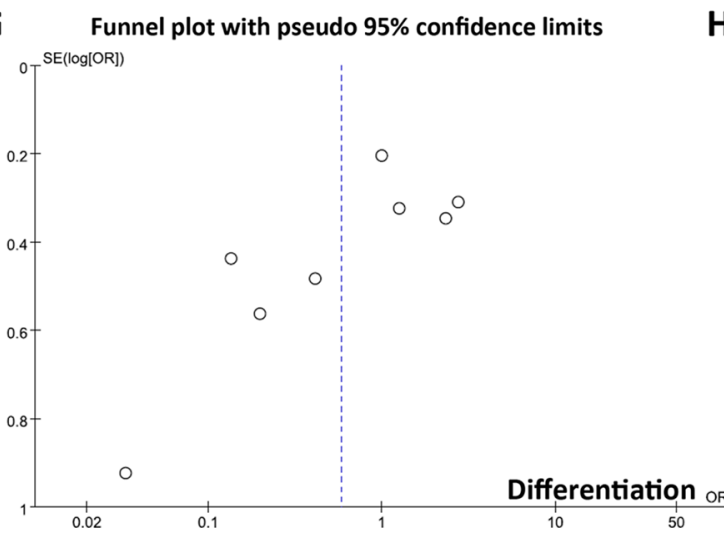

D

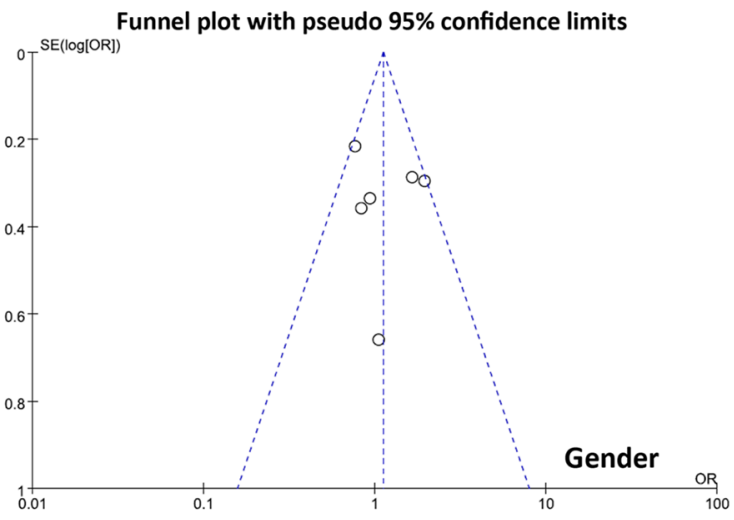

Funnel plot with pseudo 95\% confidence limits

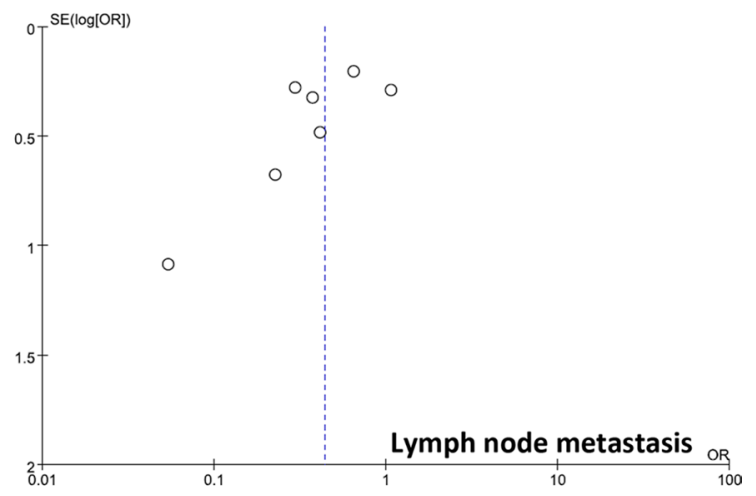

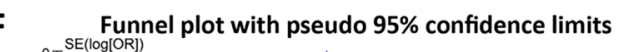

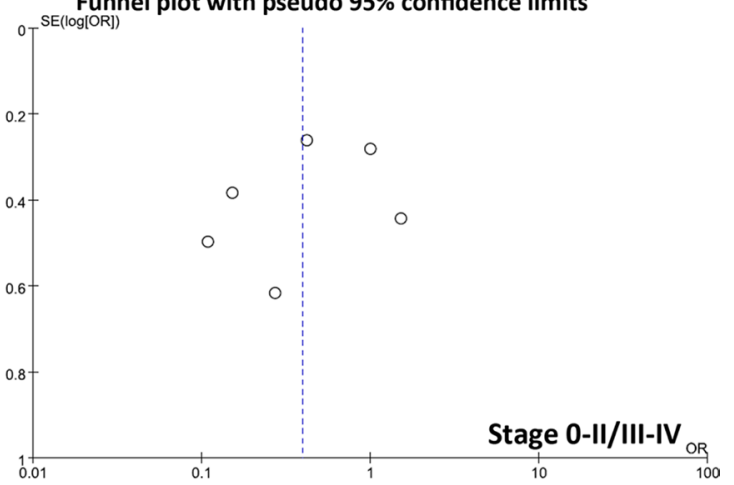

H Funnel plot with pseudo 95\% confidence limits

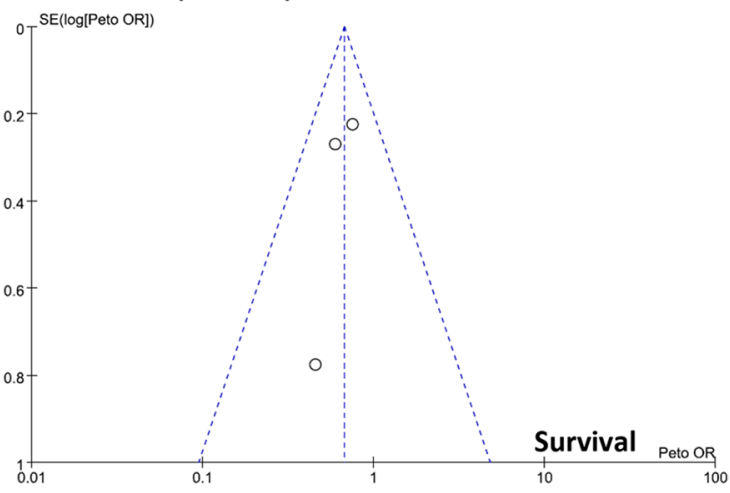

Figure 3: Funnel plot for publication bias test between fascin expression and gastric carcinogenesis or subsequent progression. The bias was analyzed about risk degrees of fascin expression in gastric mucosa for gastric carcinogenesis (A). Additionally, it was tested between fascin expression and clinicopathlogical features of gastric cancer, including age (B), depth of invasion (C), lymph node metastasis (D), TNM staging (E, F), and differentiation (G) and prognosis $(\mathbf{H})$. 
control subjects, even for early-stage ESCC. Teng et al. [33] found that the serum fascin level was markedly increased in the NSCLC patients, and positively correlated with aggressive features, including lymphatic and distant metastases. Takikita et al. [34] demonstrated that fascin expression was gradually increased from normalappearing epithelium to dysplasia to ESCC. Tsai et al. [35] showed that higher fascin immunostaining scores were significantly associated with severe dysplasia of colorectal adenomas and high-grade histopathological differentiation of colorectal adenocarcinomas. Consistent with the data about head and neck squamous cell carcinoma, lung cancer, bladder cancer, cholangiocarcinoma, urothelial carcinoma, laryngeal squamous cell carcinoma, endometrioid carcinoma, hepatocellular carcinoma (HCC), and thyroid cancer [36-44], we found up-regulated fascin expression in gastric cancer, compared with normal mucosa at both mRNA and protein levels. Moreover, fascin expression was positively associated with lymph node metastasis and TNM staging of gastric cancer. In combination with these data, it is suggested that fascin hyperexpression contributes to gastric carcinogenesis and subsequent progression.

Reportedly, fascin overexpression was associated with worse survival of the patients with lung cancer, small intestinal carcinoma, laryngeal squamous cell carcinoma, HCC, oral squamous carcinoma, breast cancer, and ESCC [29, 30, 36, 41, 43, 45, 46]. Fascin expression might be demonstrated to indicate the worse prognosis of cholangiocarcinoma, ovarian cancer, colorectal cancer, extrahepatic bile duct cancer, intrahepatic cholangiocarcinomas, or advanced ovarian serous carcinoma as an independent factor [40, 47-51]. Zhao et al. [52] found that fascin phosphorylation decreased the risk of poor survival in the ESCC patients. Teng et al. [33] demonstrated that the serum fascin level was an independent prognostic factor for M0-stage NSCLC. Our meta-analysis showed that fascin expression was positively linked to the worse prognosis of the patients with gastric cancer. Our bioinformatics data indicated
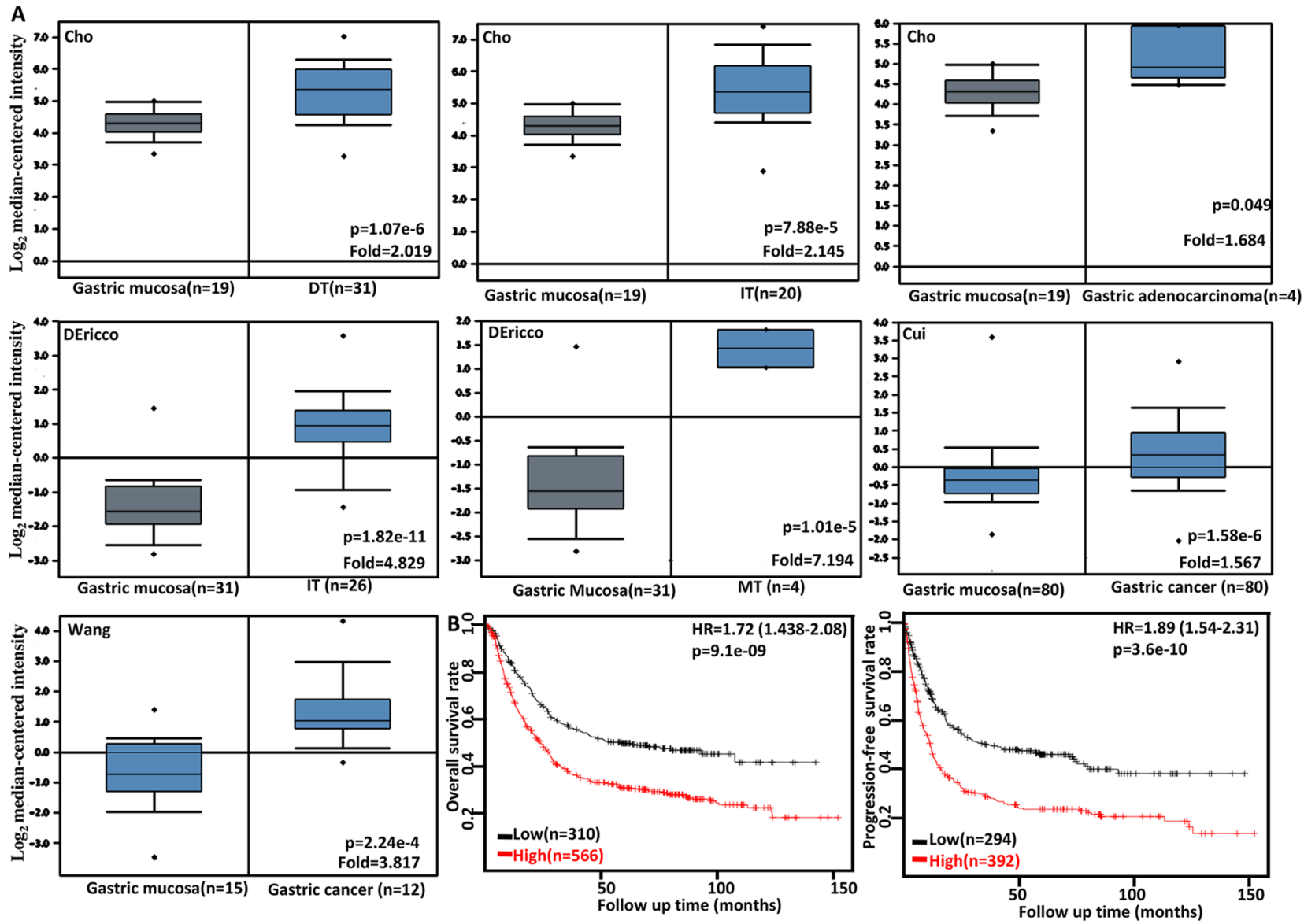

Figure 4: The clinicopathological significances of FSCN1 mRNA expression in gastric cancer. Cho's, DErrico's, Cui's and Wang's datasets were employed for bioinformatics analysis to analyze FSCN1 mRNA expression during gastric carcinogenesis. A higher FSCN1 expression was detectable in gastric cancer than that in normal gastric mucosa, even stratified into intestinal-(IT), diffuse-(DT), and mixed-type (MT) carcinomas by Lauren's classification (A, $\mathrm{p}<0.05)$. According to the data from KM plotter, FSCN1 mRNA expression was positively related to both overall and progression-free survival rates of the patients with gastric cancer (B). HR, hazard ratio. 
Table 2: The prognostic significance of FSCN1 mRNA in gastric cancer

\begin{tabular}{|c|c|c|c|c|}
\hline \multirow[t]{2}{*}{ Clinicopathological features } & \multicolumn{2}{|c|}{ Overall survival } & \multicolumn{2}{|c|}{ Progression-free survival } \\
\hline & Hazard ratio & $\mathbf{p}$ & Hazard ratio & $\mathbf{p}$ \\
\hline \multicolumn{5}{|l|}{ Sex } \\
\hline Female & $2.06(1.3-3.27)$ & 0.0017 & $2.32(1.41-3.8)$ & 0.00063 \\
\hline Male & $1.89(1.5-2.38)$ & $3 e-08$ & $2.02(1.59-2.57)$ & $6.3 e^{-}-09$ \\
\hline \multicolumn{5}{|l|}{$\mathrm{T}$} \\
\hline 2 & $2.32(1.52-3.55)$ & $6.3 e-05$ & $1.93(1.27-2.93)$ & 0.0016 \\
\hline 3 & $1.81(1.22-2.7)$ & 0.0031 & $1.69(1.15-2.48)$ & 0.0074 \\
\hline 4 & $3.4(0.79-14.59)$ & 0.081 & $4.43(1.05-18.78)$ & 0.027 \\
\hline \multicolumn{5}{|l|}{$\mathrm{N}$} \\
\hline $1-3$ & $2.02(1.54-2.66)$ & $2.9 \mathrm{e}-07$ & $2.07(1.55-2.75)$ & $3.8 \mathrm{e}-07$ \\
\hline 1 & $3.37(2.07-5.49)$ & $2.3 e-07$ & $3.01(1.89-4.8)$ & $1.1 \mathrm{e}^{-}-06$ \\
\hline 2 & $1.7(1.08-2.66)$ & 0.019 & $1.61(1.05-2.48)$ & 0.028 \\
\hline 3 & $2.32(1.33-4.07)$ & 0.0025 & $2.37(1.32-4.26)$ & 0.0028 \\
\hline \multicolumn{5}{|l|}{ M } \\
\hline 0 & $1.91(1.45-2.53)$ & $3.4 \mathrm{e}-06$ & $1.94(1.45-2.59)$ & $6 e-06$ \\
\hline 1 & - & - & - & - \\
\hline \multicolumn{5}{|l|}{ TNM staging } \\
\hline II & $2.11(1.06-4.19)$ & 0.03 & $1.89(0.95-3.76)$ & 0.067 \\
\hline III & $1.7(1.25-2.32)$ & 0.00059 & $2.09(1.41-3.09)$ & 0.00015 \\
\hline IV & $1.56(1.05-2.33)$ & 0.026 & $1.91(1.22-3)$ & 0.0042 \\
\hline \multicolumn{5}{|l|}{ Differentiation } \\
\hline Moderately-differentiated & $2.8(1.4-5.61)$ & 0.0025 & $2.53(1.31-4.89)$ & 0.0042 \\
\hline Poorly-differentiated & $1.62(0.96-2.74)$ & 0.067 & $2.16(1.34-3.47)$ & 0.0011 \\
\hline \multicolumn{5}{|l|}{ Lauren's classification } \\
\hline Intestinal-type & $2.36(1.72-3.25)$ & $5 e-08$ & $2.25(1.53-3.32)$ & $2.5 \mathrm{e}^{-}-05$ \\
\hline Diffuse-type & $1.86(1.27-2.73)$ & 0.0012 & $1.82(1.24-2.68)$ & 0.0019 \\
\hline \multicolumn{5}{|l|}{ Her2 positivity } \\
\hline- & $1.75(1.37-2.24)$ & $6.6 \mathrm{e}-06$ & $1.95(1.47-2.6)$ & $3.1 \mathrm{e}^{-}-06$ \\
\hline+ & $1.66(1.23-2.24)$ & $9 e-04$ & $2.04(1.47-2.84)$ & $1.6 \mathrm{e}-05$ \\
\hline \multicolumn{5}{|l|}{ Perforation } \\
\hline- & $1.9(1.25-2.89)$ & 0.0022 & $2.15(1.43-3.24)$ & 0.00015 \\
\hline \multicolumn{5}{|l|}{ Treatment } \\
\hline Surgery alone & $1.81(1.31-2.49)$ & 0.00022 & $1.7(1.25-2.29)$ & 0.00052 \\
\hline 5-FU-based adjuvant & $2.18(1.51-3.13)$ & $1.7 e-05$ & $2.51(1.75-3.62)$ & $3.1 \mathrm{e}^{-}-07$ \\
\hline Other adjuvant & $3.01(1.25-7.25)$ & 0.0096 & $2.9(1.32-6.39)$ & 0.0057 \\
\hline
\end{tabular}

that FSCN1 mRNA expression was negatively associated with overall and progression-free survival rates of the patient with gastric cancer, even stratified by clinicopathological features. Taken together, the mRNA and protein expression of fascin might be employed as a good and potential marker of the prognosis of the patients with gastric cancer.

In conclusion, fascin expression was upregulated in gastric cancer, and positively correlated with aggressiveness and worse prognosis of the patients with 
gastric cancer. Several limitations in our meta-analysis included the potential publication bias due from published results being predominantly positive, only Asian patient populations, subjective bias from the extracted survival data from survival curves, and small number of cancer cases in some studies.

\section{MATERIALS AND METHODS}

\section{Identification of eligible studies and data extraction}

We performed a publication search using PubMed, Web of Science, BIOSIS, SciFinder and CNKI updated on March 14, 2017. The following search terms were used: (fascin OR fscn1) AND (gastric OR stomach) AND (cancer OR carcinoma OR adenocarcinoma). Searching was done without restriction on language or publication years. Inclusion criteria for studies: (1) articles to observe the alteration in fascin expression in gastric cancer by immunohistochemistry; (2) papers to compare fascin expression with pathobiological behaviors and prognosis of gastric cancer by immunohistochemistry. Exclusion criteria included: (1) abstract, comment, review and meeting; (2) duplication of the previous publications; (3) Western blot, RT-PCR, cDNA microarray, or transcriptomic sequencing for fascin expression; (4) lack of sufficient information.

\section{Data extraction}

Based on the inclusion criteria, two reviewers (HC Zheng and S Zhao) independently extracted information from all eligible publications. The following information were included in each study: name of first author, year of publication, country, ethnicity, antibody company, numbers of cases and controls, expression alteration, and correlation with survival rate. Regarding survival analysis, we used Engauge Digitizer software to extract data from Kaplan-Meier curves and calculated the Hazard ratios (HR) and their corresponding 95\% confidence intervals (CI). Any disagreement was resolved through discussion until the two reviewers reached a consensus.

\section{Quality score assessment}

Two reviewers (HC Zheng and $\mathrm{S}$ Zhao) independently assessed the quality of the included studies according to Newcastle Ottawa Scale (NOS) (http://www. ohri.ca/programs/clinical_epidemiology/oxford.htm).

The scale consists of three components related to sample selection, comparability and ascertainment of outcome.

\section{Bioinformatics analysis}

The individual gene expression level of FSCN1 was analyzed using Oncomine (www.oncomine.org), a cancer microarray database and web-based data mining platform for a new discovery from genome-wide expression analyses. We compared the differences in FSCN1 mRNA level between gastric mucosa and cancer. All data were log-transformed, median centered per array, and standard deviation normalized to one per array. The expression (RNA-seqV2) and clinicopathological data of 392 gastric cancer patients were downloaded from the Cancer Genome Atlas (TCGA) database by TCGA-assembler in R software. We integrated the raw data, analyzed FSCN1 expression in gastric cancer, and compared it with clinicopathological and prognostic data of the patients with gastric cancer. Additionally, the prognostic significance of FSCN1 mRNA was also analyzed using Kaplan-Meier plotter (http://kmplot.com).

\section{Statistics analysis}

HWE was evaluated using Chi-square test in control groups of each study. Strength of association between fascin expression and cancer risk was assessed by odds ratios with $95 \%$ confidence intervals. Statistical significance of the pooled OR was determined by $\mathrm{Z}$ test. If there was no significant heterogeneity, the fixed effect model (MantelHaenszel method) would be employed. Otherwise, the random effect model (DerSimonian and Laird method) would be used excluding prognostic analysis. Heterogeneity effect was then quantified by $\mathrm{I}^{2}$ test, which was subdivided into low, moderate and high degrees of heterogeneity according to the cut-off values of $25 \%, 50 \%$ and $75 \%$ respectively. Publication bias was evaluated by funnel plot and quantified by Begg's test and Egger's test to assess funnel plot asymmetry. Metaanalyses were performed with Revman software 5.3 and data from TCGA database was dealt with SPSS 10.0 software using student t test. Kaplan-Meier survival plots were generated and comparisons between survival curves were made with the log-rank statistic. Two-sided $\mathrm{p}<0.05$ was considered as statistically significant.

\section{CONFLICTS OF INTEREST}

The authors have declared that no conflicts of interests exist.

\section{FUNDING}

This study was supported by Liaoning BaiQianWan Talents Program, Outstanding Scientific Fund of Shengjing Hospital, Award for Liaoning Distinguished Professor, a Key Scientific and Technological Project of Liaoning Province (2015408001) and National Natural Scientific Foundation of China (81472544; 81672700).

\section{REFERENCES}

1. Machesky LM, Li A. Fascin: Invasive filopodia promoting metastasis. Commun Integr Biol. 2010; 3:263-270. 
2. Jayo A, Parsons M. Fascin: a key regulator of cytoskeletal dynamics. Int J Biochem Cell Biol. 2010; 42:1614-1617.

3. Villari G, Jayo A, Zanet J, Fitch B, Serrels B, Frame M, Stramer BM, Goult BT, Parsons M. A direct interaction between fascin and microtubules contributes to adhesion dynamics and cell migration. J Cell Sci. 2015; 128:4601-4614

4. Lin S, Lu S, Mulaj M, Fang B, Keeley T, Wan L, Hao J, Muschol M, Sun J, Yang S. Monoubiquitination inhibits the actin bundling activity of Fascin. J Biol Chem. 2016; 291:27323-27333.

5. Sun J, He H, Xiong Y, Lu S, Shen J, Cheng A, Chang WC, Hou MF, Lancaster JM, Kim M, Yang S. Fascin protein is critical for transforming growth factor $\beta$ protein-induced invasion and filopodia formation in spindle-shaped tumor cells. J Biol Chem. 2011; 286:38865-38875.

6. Li L, Cao F, Liu B, Luo X, Ma X, Hu Z. TGF- $\beta$ induces fascin expression in gastric cancer via phosphorylation of smad3 linker area. Am J Cancer Res. 2015; 5:1890-1896.

7. Sun J, He H, Pillai S, Xiong Y, Challa S, Xu L, Chellappan $\mathrm{S}$, Yang S. GATA3 transcription factor abrogates Smad4 transcription factor-mediated fascin overexpression, invadopodium formation, and breast cancer cell invasion. J Biol Chem. 2013; 288:36971 -36982.

8. Snyder M, Huang J, Huang XY, Zhang JJ. A signal transducer and activator of transcription $3 \cdot$ Nuclear Factor $\kappa \mathrm{B}(\mathrm{Stat} 3 \cdot \mathrm{NF} \kappa \mathrm{B})$ complex is necessary for the expression of fascin in metastatic breast cancer cells in response to interleukin (IL)- 6 and tumor necrosis factor (TNF)- $\alpha$. J Biol Chem. 2014; 289:30082-30089.

9. Li D, Jin L, Alesi GN, Kim YM, Fan J, Seo JH, Wang D, Tucker M, Gu TL, Lee BH, Taunton J, Magliocca KR, Chen $\mathrm{ZG}$, et al. The prometastatic ribosomal S6 kinase 2-cAMP response element-binding protein (RSK2-CREB) signaling pathway up-regulates the actin-binding protein fascin-1 to promote tumor metastasis. J Biol Chem. 2013; 288:32528-32538.

10. Hashimoto Y, Loftis DW, Adams JC. Fascin-1 promoter activity is regulated by CREB and the aryl hydrocarbon receptor in human carcinoma cells. PLoS One. 2009; 4:e5130.

11. Osanai M, Lee GH. The retinoic acid-metabolizing enzyme CYP26A1 upregulates fascin and promotes the malignant behavior of breast carcinoma cells. Oncol Rep. 2015; 34:850-858.

12. Schoumacher M, El-Marjou F, Laé M, Kambou N, Louvard D, Robine S, Vignjevic DM. Conditional expression of fascin increases tumor progression in a mouse model of intestinal cancer. Eur J Cell Biol. 2014; 93:388-395.

13. Li A, Morton JP, Ma Y, Karim SA, Zhou Y, Faller WJ, Woodham EF, Morris HT, Stevenson RP, Juin A, Jamieson NB, MacKay CJ, Carter CR, et al. Fascin is regulated by slug, promotes progression of pancreatic cancer in mice, and is associated with patient outcomes. Gastroenterology. 2014; 146:1386-1396.
14. Kulasingam V, Diamandis EP. Fascin-1 is a novel biomarker of aggressiveness in some carcinomas. BMC Med. 2013; 11:53.

15. Tan VY, Lewis SJ, Adams JC, Martin RM. Association of fascin-1 with mortality, disease progression and metastasis in carcinomas: a systematic review and meta-analysis. BMC Med. 2013; 11:52.

16. Gan FL, Wu QT, Hao XC, Li Y. Fascin expression in gastric cancer. Med Equip. 2013; 26:36.

17. Li K, Wang B, Feng GS. Expression of fascin, nm23 and c-erbB-2 in gastric carcinoma and its clinical significance. Chi Arch Gen Surg. 2009; 3:14-18.

18. Li M, Liu ZP. The relationship between Fascin and SFRP1 expression in gastric cancer. Heilongjiang Med and Pharm. 2014; 37:97-98.

19. Li S, Cai L, Zhang RQ, Zhang N, Zhou Y, Xu YQ. The correlation between expression of fascin and mutation of K-ras in gastric cancer tissue. Prog Mod Biomed. 2015; 15:4014-4016.

20. Li XH, Zheng H, Hara T, Takahashi H, Masuda S, Wang Z, Yang X, Guan Y, Takano Y. Aberrant expression of cortactin and fascin are effective markers for pathogenesis, invasion, metastasis and prognosis of gastric carcinomas. Int J Oncol. 2008; 33:69-79.

21. Peng LT. The expression of clinical significance of Fascin in gastric carcinoma and adjacent mucosa. Proceed Clin Med. 2011; 20:520-521.

22. Tu L, Xu J, Wang M, Zhao WY, Zhang ZZ, Zhu CC, Tang DF, Zhang YQ, Wang DH, Zuo J, Cao H. Correlations of fascin-1 and cadherin-17 protein expression with clinicopathologic features and prognosis of patients with gastric cancer. Tumour Biol. 2016; 37:8775-8782.

23. Hashimoto Y, Shimada Y, Kawamura J, Yamasaki S, Imamura $\mathrm{M}$. The prognostic relevance of fascin expression in human gastric carcinoma. Oncology. 2004; 67:262-70.

24. Kim SJ, Choi IJ, Cheong TC, Lee SJ, Lotan R, Park SH, Chun KH. Galectin-3 increases gastric cancer cell motility by up-regulating fascin-1 expression. Gastroenterology. 2010; 138:1035-1045.

25. Rao P, Li GC, Xu LP, Zhou HC, Liu JC, Peng GX, He F, Liu X, Liu ZH, Wang ZQ. Expression of Runx3 and fascin and clinical significance in gastric cancer. Prac Clin Med. 2013; 14:10-11.

26. Tsai WC, Jin JS, Chang WK, Chan DC, Yeh MK, Cherng SC, Lin LF, Sheu LF, Chao YC. Association of cortactin and fascin-1 expression in gastric adenocarcinoma:correlation with clinicopathological parameters. J Histochem Cytochem. 2007; 55:955-962.

27. Zhao H, Yang F, Zhao W, Zhang C, Liu J. Fascin overexpression promotes cholangiocarcinoma RBE cell proliferation, migration, and invasion. Technol Cancer Res Treat. 2016; 15:322-333.

28. Darnel AD, Behmoaram E, Vollmer RT, Corcos J, Bijian K, Sircar K, Su J, Jiao J, Alaoui-Jamali MA, Bismar TA. Fascin 
regulates prostate cancer cell invasion and is associated with metastasis and biochemical failure in prostate cancer. Clin Cancer Res. 2009; 15:1376-1383.

29. Al-Alwan M, Olabi S, Ghebeh H, Barhoush E, Tulbah A, Al-Tweigeri T, Ajarim D, Adra C. Fascin is a key regulator of breast cancer invasion that acts via the modification of metastasis-associated molecules. PLoS One. 2011; 6:e27339.

30. Alam H, Bhate AV, Gangadaran P, Sawant SS, Salot S, Sehgal L, Dange PP, Chaukar DA, D'cruz AK, Kannanl S, Gude R, Kane S, Dalal SN, Vaidya MM. Fascin overexpression promotes neoplastic progression in oral squamous cell carcinoma. BMC Cancer. 2012; 12:32.

31. Liang Z, Wang Y, Shen Z, Teng X, Li X, Li C, Wu W, Zhou $Z$, Wang $Z$. Fascin 1 promoted the growth and migration of non-small cell lung cancer cells by activating YAP/TEAD signaling. Tumour Biol. 2016; 37:10909-10915.

32. Chen WX, Hong XB, Hong CQ, Liu M, Li L, Huang LS, Xu LY, Xu YW, Peng YH, Li EM. Tumor-associated autoantibodies against Fascin as a novel diagnostic biomarker for esophageal squamous cell carcinoma. Clin Res Hepatol Gastroenterol. 2016; 41: 327-332.

33. Teng Y, Xu S, Yue W, Ma L, Zhang L, Zhao X, Guo Y, Zhang C, Gu M, Wang Y. Serological investigation of the clinical significance of fascin in non-small-cell lung cancer. Lung Cancer. 2013; 82:346-352.

34. Takikita M,Hu N, Shou JZ, Giffen C, Wang QH, Wang C, Hewitt SM, Taylor PR. Fascin and CK4 as biomarkers for esophageal squamous cell carcinoma. Anticancer Res. 2011; 31:945-952.

35. Tsai WC, Chao YC, Sheu LF, Chang JL, Nieh S, Jin JS. Overexpression of fascin-1 in advanced colorectal adenocarcinoma: tissue microarray analysis of immunostaining scores with clinicopathological parameters. Dis Markers. 2007; 23:153-160.

36. Ling XL, Zhang T, Hou XM, Zhao D. Clinicopathological significance of fascin-1 expression in patients with non-small cell lung cancer. Onco Targets Ther. 2015; 8:1589-1595.

37. Papaspyrou K, Brochhausen C, Schmidtmann I, Fruth K, Gouveris H, Kirckpatrick J, Mann W, Brieger J. Fascin upregulation in primary head and neck squamous cell carcinoma is associated with lymphatic metastasis. Oncol Lett. 2014; 7:2041-2046.

38. El-Rehim DM, El-Maqsoud NM, El-Hamid AM, El-Bab TK, Galal EM. Expression of extracellular matrix metalloproteinase inducer and fascin in urinary bladder cancer: Correlation with clinicopathological characteristics. Mol Clin Oncol. 2013;1:297-304.

39. Bi JB, Zhu Y, Chen XL, Yu M, Zhang YX, Li BX, Sun JW, Shen HL, Kong CZ. The role of fascin in migration and invasion of urothelial carcinoma of the bladder. Urol Int. 2013; 91:227-235.

40. Mao X, Chen D, Wu J, Li J, Zhou H, Wu Y, Duan X. Differential expression of fascin, E-cadherin and vimentin:
Proteins associated with survival of cholangiocarcinoma patients. Am J Med Sci. 2013; 346:261-268.

41. Gao W, Zhang C, Feng Y, Chen G, Wen S, Huangfu H, Wang B. Fascin-1, ezrin and paxillin contribute to the malignant progression and are predictors of clinical prognosis in laryngeal squamous cell carcinoma. PLoS One. 2012; 7:e50710.

42. Gun BD, Bahadir B, Bektas S, Barut F, Yurdakan G, Kandemir NO, Ozdamar SO. Clinicopathological significance of fascin and CD44v6 expression in endometrioid carcinoma. Diagn Pathol. 2012; 7:80.

43. Huang X, Ji J, Xue H, Zhang F, Han X, Cai Y, Zhang J, Ji G. Fascin and cortactin expression is correlated with a poor prognosis in hepatocellular carcinoma. Eur J Gastroenterol Hepatol. 2012; 24:633-639.

44. Chen G, Zhang FR, Ren J, Tao LH, Shen ZY, Lv Z, Yu SJ, Dong BF, Xu LY, Li EM. Expression of fascin in thyroid neoplasms: a novel diagnostic marker. J Cancer Res Clin Oncol. 2008; 134:947-951.

45. Gu MJ, Kim JY, Park JB. Fascin expression predicts lymph node metastasis and worse survival in small intestinal carcinoma. Pathology. 2014; 46:21-24.

46. Hsu KF, Lin CK, Yu CP, Tzao C, Lee SC, Lee YY, Tsai WC, Jin JS. Cortactin, fascin, and survivin expression associated with clinicopathological parameters in esophageal squamous cell carcinoma. Dis Esophagus. 2009; 22:402-408.

47. Hanker LC, Karn T, Holtrich U, Graeser M, Becker S, Reinhard J, Ruckhäberle E, Gevensleben H, Rody A. Prognostic impact of fascin-1 (FSCN1) in epithelial ovarian cancer. Anticancer Res. 2013; 33:371-377.

48. Jung EJ, Lee JH, Min BW, Kim YS, Choi JS. Clinicopathologic significance of fascin, extracellular matrix metalloproteinase inducer, and ezrin expressions in colorectal adenocarcinoma. Indian J Pathol Microbiol. 2011; 54:32-36.

49. Won KY, Kim GY, Lim SJ, Park YK, Kim YW. Prognostic significance of fascin expression in extrahepatic bile duct carcinomas. Pathol Res Pract. 2009; 205:742-748.

50. Iguchi T, Aishima S, Taketomi A, Nishihara Y, Fujita N, Sanefuji K, Sugimachi K, Yamashita Y, Maehara Y, Tsuneyoshi M. Fascin overexpression is involved in carcinogenesis and prognosis of human intrahepatic cholangiocarcinoma: immunohistochemical and molecular analysis. Hum Pathol. 2009; 40:174-180.

51. Daponte A, Kostopoulou E, Papandreou CN, Daliani DD, Minas M, Koukoulis G, Messinis IE. Prognostic significance of fascin expression in advanced poorly differentiated serous ovarian cancer. Anticancer Res. 2008; 28:1905-1901.

52. Zhao Q, Shen JH, Shen ZY, Wu ZY, Xu XE, Xie JJ, Wu JY, Huang Q, Lu XF, Li EM, Xu LY. Phosphorylation of fascin decreases the risk of poor survival in patients with esophageal squamous cell carcinoma. J Histochem Cytochem. 2010; 58:979-988. 